\title{
Nonlinear response of permanent dipoles in a uniaxial potential to alternating fields
}

\author{
W. T. Coffey, ${ }^{1}$ D. S. F. Crothers, ${ }^{2}$ Yu. P. Kalmykov, ${ }^{3}$ and P. M. Déjardin ${ }^{3}$ \\ ${ }^{1}$ Department of Electronic and Electrical Engineering, Trinity College, Dublin 2, Ireland \\ ${ }^{2}$ Department of Applied Mathematics and Theoretical Physics, The Queens University of Belfast, Belfast BT7 1NN, Northern Ireland \\ ${ }^{3}$ MEPS, Université de Perpignan, 52 Avenue Paul Alduy, 66860 Perpignan Cedex, France \\ (Received 10 January 2005; revised manuscript received 16 March 2005; published 23 June 2005)
}

\begin{abstract}
It is shown how the existing theory of the dynamic Kerr effect and nonlinear dielectric relaxation based on the noninertial Brownian rotation of noninteracting rigid dipolar particles may be generalized to take into account interparticle interactions using the Maier-Saupe mean field potential. The results (available in simple closed form) suggest that the frequency dependent nonlinear response provides a method of measuring the Kramers escape rate (or in the analogous problem of magnetic relaxation of fine single domain ferromagnetic particles, the superparamagnetic relaxation time).
\end{abstract}

DOI: 10.1103/PhysRevE.71.062102

A system in thermal equilibrium at temperature $T$ disturbed by an external stimulus evolves to a new equilibrium (stationary) state. Moreover, if the energy stimulus is much lower than the thermal energy $k_{B} T$, linear (in the stimulus) deviations of the expectation value of the relevant dynamical variable in the stationary state are sufficient to evaluate the generalized susceptibility (linear ac response) using appropriate equilibrium (stationary) correlation functions. The calculation of the nonlinear stationary (ac) response even for systems of noninteracting particles with a single coordinate is, however, much more difficult because no connection between the transient and the ac responses exists. If interactions are included the difficulties are compounded. Nonlinear dielectric relaxation and the dynamic Kerr effect of permanent dipoles in a mean field potential are naturally occurring examples.

In this context we remark that the orientational electric polarization of noninteracting permanent dipoles in an ac field $\mathbf{E}(t)$ treated by Debye [1] depends in the linear approximation in $\mathbf{E}(t)$ on the average over orientations of the Legendre polynomial $\left\langle P_{1}(\cos \vartheta)\right\rangle(t), \vartheta$ being the polar angle of the electric dipole moment vector $\boldsymbol{\mu}$. Similar remarks apply to the magnetization of blocked noninteracting ferrofluid particles with magnetic dipole moment $\boldsymbol{\mu}$ in ac magnetic fields $\mathbf{H}(t)$ [2]. Subsequently [2-6] Debye's calculation was generalized to nonlinear responses. We mention $\left\langle P_{2}\right\rangle(t)$ governing the Kerr effect response (KER) [3-5] and the nonlinear dielectric effect (NLDE) [6,7] amending $\left\langle P_{1}\right\rangle(t)$ to $O\left(E^{3}\right)$. The conclusions are [to $O\left(E^{2}\right)$ ] for the KER for a pure sinusoid that the square law nonlinearity rectifies $\mathbf{E}(t)$ yielding a frequency dependent dc response superimposed on which is the dephased second harmonic [8]. In the NLDE, additional terms in the fundamental and in the third harmonic appear in $\left\langle P_{1}\right\rangle(t)$. Experimental confirmation has been reported [7,9]. The Debye theory may not be used for dense anisotropic dipolar systems, where intermolecular interactions occur, such as nematic liquid crystals. Here dielectric relaxation is usually interpreted using as model the noninertial rotational Brownian motion of a rodlike particle in an external potential $V$ (e.g., [6,10-12]). This model was used in Ref. [13], where the exact linear ac response is calculated in terms of continued fractions (using linear response theory [14]) for the Maier-Saupe uniaxial anisotropy potential
PACS number(s): 05.40.- a, 77.22.-d, 05.10.Gg, 78.20.Jq

$$
V=-K \cos ^{2} \vartheta
$$

where $K$ is the anisotropy constant. Exact solutions for the nonlinear ac response in a uniaxial potential can also be obtained by matrix continued fractions without using perturbation theory $[5,6]$. However, that approach cannot yield simple formulas for experimental comparison and, moreover, it cannot provide an exact evolution equation for the ac responses for perturbation purposes. Preliminary steps toward this were made in Refs. [2-6,15-18] for dielectric relaxation of dipolar systems and for magnetic relaxation (superparamagnetism) of fine single domain ferromagnetic particles (in most respects a replica of dielectric relaxation of nematics). Here we demonstrate how by calculating from perturbation theory the linear ac response in the presence of $\mathbf{E}(t)$ one may generate the KER and all higher order nonlinear responses. The linear response comprising an infinity of relaxation modes may be accurately represented by two modes, that of low frequency arising from the slow barrier crossing of dipoles and that of high frequency representing the infinity of fast near-degenerate "intrawell" modes approximated as a single high frequency mode. The analytical responses are obtained utilizing the two-mode approximation for linear response combined with Morita's treatment [19] of nonlinear response, showing how the distribution function induced by a strong perturbing field may be calculated from the Green functions in the absence of the perturbation, with linear response theory as a special case.

The cornerstone of our calculation is the Smoluchowski (Fokker-Planck) equation for the density $W(\boldsymbol{\mu}, t)$ of orientations of dipoles $\boldsymbol{\mu}$ on the surface of the unit sphere $[1,4-6]$

$$
\dot{W}=\left[L_{F P}+L_{e x t}(t)\right] W,
$$

where $L_{F P} W=\left(2 \tau_{D}\right)^{-1}[\Delta W+\beta \boldsymbol{\nabla} \cdot(W \boldsymbol{\nabla} V)]$ is the unperturbed Fokker-Planck operator while $L_{\text {ext }} W=\left(2 \tau_{D}\right)^{-1} \beta \boldsymbol{\nabla} \cdot\left(W \boldsymbol{\nabla} V_{\text {ext }}\right)$ is the Zeeman energy $V_{\text {ext }}=-(\mathbf{E} \cdot \boldsymbol{\mu})$ contribution, and $\boldsymbol{\nabla}$ and $\Delta$ are the gradient and Laplacian on the surface of the unit sphere. Here $\beta=\left(k_{B} T\right)^{-1}, \tau_{D}=\beta \zeta / 2$ is the Debye relaxation time for free diffusion, and $\zeta$ is the viscous drag coefficient. Expanding $W$ in the $\left\{P_{n}\right\}$ yields [4-6] 


$$
\begin{aligned}
& \tau_{D} \dot{f}_{n}(t)+c_{n} f_{n-2}(t)+d_{n} f_{n}(t)+g_{n} f_{n+2}(t) \\
& =\xi(t) a_{n}\left[f_{n-1}(t)-f_{n+1}(t)\right]
\end{aligned}
$$

where $f_{n}(t)=\left\langle P_{n}(\cos \vartheta)\right\rangle(t)$ and $\xi(t)=\beta \mu E(t)$, and all the coefficients are given, e.g., in Refs. [4-6]. One may write

$$
f_{n}(t)=f_{n}^{(0)}+f_{n}^{(1)}(t)+f_{n}^{(2)}(t)+f_{n}^{(3)}(t)+\cdots
$$

[with the superscripts denoting the relevant order in $\mathbf{E}(t)$ ] so that

$$
\begin{aligned}
& \tau_{D} \dot{f}_{n}^{(m)}(t)+c_{n} f_{n-2}^{(m)}(t)+d_{n} f_{n}^{(m)}(t)+g_{n} f_{n+2}^{(m)}(t) \\
& =\xi(t) a_{n}\left[f_{n-1}^{(m-1)}(t)-f_{n+1}^{(m-1)}(t)\right] .
\end{aligned}
$$

Thus to calculate the matrix element $f_{2}^{(2)}(t)$, i.e., the lowest order approximation to the KER, we first determine $\left\{f_{2 n-1}^{(1)}(t)\right\}$ satisfying Eq. (3) with $m=1$. The exact solutions of Eq. (3) for $f_{2 n-1}^{(1)}(t)$ for the stationary response to $\xi(t)=\xi e^{i \omega t}$ are given by continued fractions [5]. However, in order to obtain analytical approximations, we use another method. Suppose that a small probing field $\xi_{1}=\beta \mu E_{1} \ll 1$ applied along the polar axis at $t=-\infty$ is removed at $t=0$. The step-off $[\xi(t)=0$ for $t>0]$ solution of Eq. (3) for $m=1 f_{2 n-1, o f f}^{(1)}(t)$ is

$$
f_{2 n-1, o f f}^{(1)}(t)=\xi_{1} \chi_{2 n-1} \Phi_{1,2 n-1}(t),
$$

where $\Phi_{1,2 n-1}(t)$ are the normalized equilibrium correlation functions defined as

$$
\Phi_{k, m}(t)=\frac{\left\langle P_{k}[\cos \vartheta(0)] P_{m}[\cos \vartheta(t)]\right\rangle_{0}-\left\langle P_{k}\right\rangle_{0}\left\langle P_{m}\right\rangle_{0}}{\left\langle P_{k} P_{m}\right\rangle_{0}-\left\langle P_{k}\right\rangle_{0}\left\langle P_{m}\right\rangle_{0}},
$$

$\left\langle P_{n}\right\rangle_{0}=\left\langle P_{n}[\cos \vartheta(0)]\right\rangle_{0}$, and $\chi_{2 n-1}=\left\langle P_{1} P_{2 n-1}\right\rangle_{0}$ are the static susceptibilities, which can be expressed as hypergeometric functions [13]. The Green functions $G_{2 n-1}(t)$ of the unperturbed $[\xi(t)=0]$ Eq. (3) with $m=1$ is $G_{2 n-1}(t)=-\dot{\Phi}_{1,2 n-1}(t)$ [14]. Thus

$$
f_{2 n-1}^{(1)}(t)=-\chi_{2 n-1} \int_{-\infty}^{t} \dot{\Phi}_{1,2 n-1}\left(t-t^{\prime}\right) \xi\left(t^{\prime}\right) d t^{\prime} .
$$

If $\xi(t)=\xi e^{i \omega t}$, Eq. (6) yields $f_{2 n-1}^{(1)}(t)=\chi_{2 n-1}(\omega) \xi e^{i \omega t}$, where $\chi_{2 n-1}(\omega)$ are the generalized complex susceptibilities

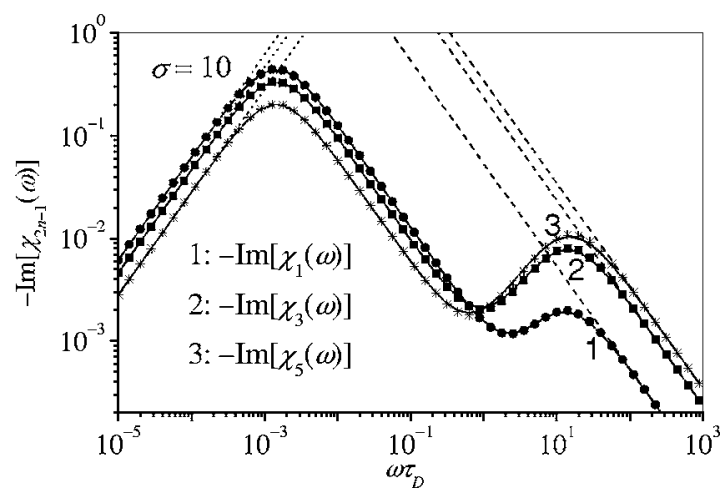

FIG. 1. $-\operatorname{Im}\left[\chi_{2 n-1}(\omega)\right]$ vs $\omega \tau_{D}$ [solid lines, Eqs. (3) and (7) for $n=1,3,5] ; \chi_{2 n-1} \omega \tau_{2 n-1}$ (dotted lines); and $\chi_{2 n-1}\left(\omega \tau_{2 n-1}^{\text {eff }}\right)^{-1}$ (dashed lines). Symbols, Eq. (9)

$$
\frac{\chi_{2 n-1}(\omega)}{\chi_{2 n-1}}=1-i \omega \int_{0}^{\infty} \Phi_{1,2 n-1}(t) e^{-i \omega t} d t .
$$

The time domain behavior of $\Phi_{1,2 n-1}(t)$ is characterized by the integral and effective relaxation times

$$
\tau_{2 n-1}=\int_{0}^{\infty} \Phi_{1,2 n-1}(t) d t, \quad \tau_{2 n-1}^{e f f}=-1 / \dot{\Phi}_{1,2 n-1}(0) .
$$

Here $\tau_{2 n-1}^{e f f}$ is evaluated from Eq. (3) with $\xi(t)=0$ using equilibrium averages as

$$
\begin{aligned}
\frac{\tau_{2 n-1}^{e f f}}{\tau_{D}} & =-\frac{f_{2 n-1, o f f}^{(1)}(0)}{\tau_{D} \dot{f}_{2 n-1, o f f}^{(1)}(0)} \\
& =\left\{d_{2 n-1}+c_{2 n-1} \frac{\left\langle P_{1} P_{2 n-3}\right\rangle_{0}}{\left\langle P_{1} P_{2 n-1}\right\rangle_{0}}+g_{2 n-1} \frac{\left\langle P_{1} P_{2 n+1}\right\rangle_{0}}{\left\langle P_{1} P_{2 n-1}\right\rangle_{0}}\right\}^{-1}
\end{aligned}
$$

and $\tau_{2 n-1}$ is given by the mean first passage time approach of Szabo [20], which for the present problem yields

$$
\begin{aligned}
\tau_{2 n-1}= & \frac{2 \tau_{D}}{Z\left\langle P_{1} P_{2 n-1}\right\rangle_{0}} \int_{-1}^{1} \frac{e^{-\sigma z^{2}}}{1-z^{2}} \int_{-1}^{z} x e^{\sigma x^{2}} d x \\
& \times \int_{-1}^{z} P_{2 n-1}(y) e^{\sigma y^{2}} d y d z,
\end{aligned}
$$

where $Z=\int_{-1}^{1} e^{\sigma z^{2}} d z$ is the partition function and $\sigma=\beta K$ is the barrier height parameter. $\Phi_{1,2 n-1}(t)$ may also be written as an eigensolution using the eigenvalues $\left\{\lambda_{k}\right\}$ of $L_{F P}$, viz., $\Phi_{1,2 n-1}(t)=\Sigma_{k} c_{k}^{n} e^{-t \lambda_{k}}$, where $\Sigma_{k} c_{k}^{n}=1$ and $\lambda_{1}$ (essentially the Kramers escape rate) is associated with the slowest relaxation mode and so with the long time behavior of $\Phi_{1,2 n-1}(t)$; the other $\lambda_{k}$ characterize high frequency intrawell modes. By Eq. (8) $\tau_{2 n-1}=\Sigma_{k} c_{k}^{n} / \lambda_{k}$ and $\tau_{2 n-1}^{e f f}=1 / \Sigma_{k} c_{k}^{n} \lambda_{k}$. The behavior of $\lambda_{1}, \tau_{2 n-1}$, and $\tau_{2 n-1}^{e f f}$ is given, for $\sigma \leqslant 1$, by

$$
\begin{gathered}
\lambda_{1} \tau_{D}=1-\frac{2}{5} \sigma+\cdots, \\
\frac{\tau_{2 n-1}}{\tau_{D}}=\frac{n !-(1 / 2)_{n}}{n(1 / 2)_{n}}+\sigma \frac{2+8 n ! /(3 / 2)_{n-2}}{3 n(4 n+1)}+\cdots, \\
\frac{\tau_{2 n-1}^{e f f}}{\tau_{D}}=\frac{1}{n}+\frac{2 \sigma}{4 n^{2}+n}+\ldots
\end{gathered}
$$

$[(a)]$ is the Pochhammer symbol] and, for $\sigma \gg 1$, by

$$
\begin{gathered}
\lambda_{1} \tau_{D} \sim \frac{2 \sigma^{3 / 2} e^{-\sigma}}{\sqrt{\pi}}\left(1-\frac{1}{\sigma}-\frac{3}{4 \sigma^{2}}+\ldots\right), \\
\frac{\tau_{2 n-1}}{\tau_{D}} \sim \frac{\sqrt{\pi} e^{\sigma}}{2 \sigma^{3 / 2}}\left(1+\frac{1}{\sigma}+\frac{7+n-2 n^{2}}{4 \sigma^{2}}+\cdots\right), \\
\frac{\tau_{2 n-1}^{e f f}}{\tau_{D}} \sim \frac{2 \sigma}{2 n^{2}-n}\left(1-\frac{3}{2 \sigma}+\cdots\right) .
\end{gathered}
$$

The spectra of $\chi_{2 n-1}(\omega)$ can be accurately described at all frequencies (see Fig. 1) by a sum of two Lorentzians, viz., 
TABLE I. Numerical values of $\left(\lambda_{1} \tau_{D}\right)^{-1}, \tau^{e f f} / \tau_{D}$, and $\tau / \tau_{D}$.

\begin{tabular}{cccccccccc}
\hline \hline$\sigma$ & 0 & 1 & 2 & 3 & 4 & 5 & 6 & 8 & 10 \\
\hline$\left(\lambda_{1} \tau_{D}\right)^{-1}$ & 1.0 & 1.531 & 2.476 & 4.243 & 7.702 & 14.77 & 29.75 & 135.8 & 693.9 \\
$\tau^{e f f} / \tau_{D}$ & -1.0 & -2.169 & 83.37 & 2.411 & 1.352 & 1.021 & 0.881 & 0.805 & 0.826 \\
$\tau / \tau_{D}$ & 1.0 & 1.582 & 2.655 & 4.713 & 8.788 & 17.09 & 34.43 & 153.2 & 757.9 \\
\hline \hline
\end{tabular}

$$
\frac{\chi_{2 n-1}(\omega)}{\chi_{2 n-1}}=\frac{\Delta_{2 n-1}}{1+i \omega / \lambda_{1}}+\frac{1-\Delta_{2 n-1}}{1+i \omega \tau_{W}^{2 n-1}},
$$

where $\Delta_{2 n-1}$ and $\tau_{W}^{2 n-1}$ are determined so as to ensure the correct low and high frequency behavior of $\chi_{2 n-1}(\omega)$, viz., $\chi_{2 n-1}(\omega) / \chi_{2 n-1} \approx 1-i \omega \tau_{2 n-1}$ as $\omega \rightarrow 0$ and $\chi_{2 n-1} / \chi_{2 n-1}(\omega)$ $\sim i \omega \tau_{2 n-1}^{e f f}$ as $\omega \rightarrow \infty$, and are given by

$$
\Delta_{m}=\frac{\tau_{m} / \tau_{m}^{\text {eff }}-1}{\lambda_{1} \tau_{m}-2+1 /\left(\lambda_{1} \tau_{m}^{e f f}\right)}, \quad \tau_{W}^{m}=\frac{\lambda_{1} \tau_{m}-1}{\lambda_{1}-1 / \tau_{m}^{e f f}} .
$$

In the time domain, the two-mode approximation Eq. (9) is equivalent to assuming that the relaxation function $\Phi_{1,2 n-1}(t)$ (which in general comprises an infinite number of exponentials) may be approximated by two exponentials only. An interested reader can find a detailed description and various applications of this two-mode approximation in Ref. [6].

The second order response $\left\{f_{2 n}^{(2)}(t)\right\}$ satisfies Eq. (3) with $m=2$. The exact solution for the element $f_{2}^{(2)}(t)$ governing the $\mathrm{KER}$, with $\xi(t)=\xi \cos \omega t$, is

$$
f_{2}^{(2)}(t)=\xi^{2} \operatorname{Re}\left[F_{0}^{(2)}(\omega)+F_{2}^{(2)}(\omega) e^{2 i \omega t}\right]
$$

where the frequency dependent dc $F_{0}^{(2)}(\omega)$ and the second harmonic $F_{2}^{(2)}(\omega)$ terms are

$$
\begin{aligned}
\left(\begin{array}{c}
F_{0}^{(2)}(\omega) \\
F_{2}^{(2)}(\omega)
\end{array}\right)= & \frac{3 \sqrt{\pi}}{4 \sigma} \sum_{n=1}^{\infty} \frac{(-1)^{n+1} n !}{\Gamma(n+1 / 2)} \prod_{k=1}^{n}\left(\begin{array}{c}
S_{2 k}(0) \\
S_{2 k}(2 i \omega)
\end{array}\right) \\
& \times\left[\chi_{2 n-1}(\omega)-\chi_{2 n+1}(\omega)\right] .
\end{aligned}
$$

and the continued fractions $S_{n}(i \omega)$ are defined as $S_{n}(i \omega)$ $=c_{n}\left[i \omega \tau_{D}-d_{n}-g_{n} S_{n+2}(i \omega)\right]^{-1}[\mathrm{cf}$. [13], Eq. (26)]. In order to obtain a simple analytic approximation for the KER, we notice that the normalized step-off solution of Eq. (3) with $m$ $=2$ is $f_{2, o f f}^{(2)}(t)=\xi^{2} \chi_{2} \Phi_{2,2}(t)$, where $\Phi_{2,2}(t)$ is the normalized

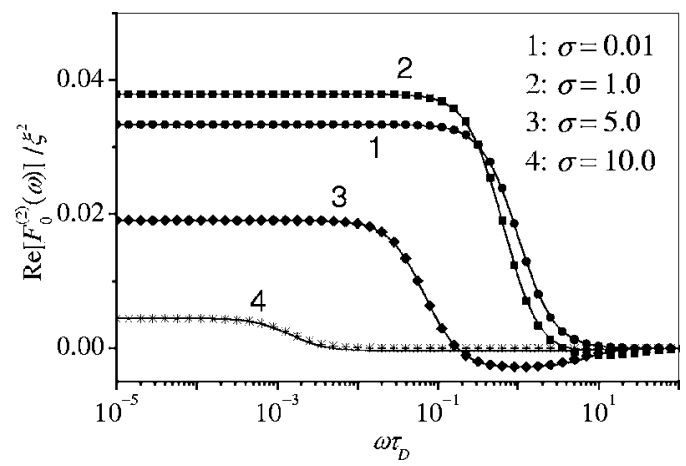

FIG. 2. Exact $\operatorname{Re}\left[F_{0}^{(2)}(\omega)\right] / \xi^{2}$ [Eq. (12), solid lines] and approximate [Eq. (16), symbols] solutions. second rank equilibrium correlation function defined by Eq. (5) and $\chi_{2}=\left(\left\langle P_{2}^{2}\right\rangle_{0}-\left\langle P_{2}\right\rangle_{0}^{2}\right) / 3$. As the overbarrier relaxation mode is not involved in the propagator of $f_{2}^{(2)}(t)$, one may use a single mode approximation for $\Phi_{2,2}(t)$, viz.,

$$
\Phi_{2,2}(t) \approx e^{-t / \tau_{2}^{e f f}},
$$

with the effective relaxation time $\tau_{2}^{e f f}$ given by

$$
\frac{\tau_{2}^{e f f}}{\tau_{D}}=-\frac{f_{2, o f f}^{(2)}(0)}{\tau_{D} \dot{f}_{2, o f f}^{(2)}(0)}=\frac{\left\langle P_{2}^{2}\right\rangle_{0}-\left\langle P_{2}\right\rangle_{0}^{2}}{1+\left\langle P_{2}\right\rangle_{0}-2\left\langle P_{2}^{2}\right\rangle_{0}} \text {. }
$$

The qualitative behavior of $\tau_{2}^{\text {eff }}$ is $\tau_{2}^{\text {eff }} / \tau_{D}=1 / 3+2 \sigma / 189$ $+\cdots$ for $\sigma \leqslant 1$ and $\tau_{2}^{\text {eff }} / \tau_{D}=\sigma^{-1} / 2+\sigma^{-2} 5 / 4+\cdots$ for $\sigma \gg 1$. Moreover, using the effective relaxation time means that Eq. (3) for $m=2$ can be represented as

$$
\tau_{2}^{e f f} \dot{f}_{2}^{(2)}(t)+f_{2}^{(2)}(t)=-\chi_{2} \xi(t) \int_{-\infty}^{t} \dot{\Phi}^{(1)}\left(t-t^{\prime}\right) \xi\left(t^{\prime}\right) d t^{\prime}
$$

with solution

$$
f_{2}^{(2)}(t)=-\frac{\chi_{2}}{\tau_{2}^{e f}} \int_{-\infty}^{t} \xi\left(t^{\prime}\right) e^{-\left(t-t^{\prime}\right) / \tau_{2}^{e f}} \int_{-\infty}^{t^{\prime}} \dot{\Phi}^{(1)}\left(t^{\prime}-t^{\prime \prime}\right) \xi\left(t^{\prime \prime}\right) d t^{\prime \prime} d t^{\prime} .
$$

Here $\Phi^{(1)}(t)$ is the normalized $\left[\Phi^{(1)}(0)=1\right]$ effective relaxation function, accounting for the driving functions $\left\{f_{2 n-1}^{(1)}(t)\right\}$. As before $\Phi^{(1)}(t)$ is characterized by the integral, $\tau$, and effective, $\tau^{e f f}$, relaxation times, which can be estimated from the low and high frequency asymptotes of the dc KER $\tau=-\lim _{\omega \rightarrow 0} 2 \operatorname{Im}\left[F_{0}^{(2)}(\omega)\right] /\left(\omega \chi_{2}\right)$ and $\tau^{\text {eff }}$ $=\lim _{\omega \rightarrow \infty} \chi_{2}\left\{2 \omega \operatorname{Im}\left[F_{0}^{(2)}(\omega)\right]\right\}^{-1}$. The one-sided Fourier transform of $-\dot{\Phi}^{(1)}(t)$ may be represented in a two-mode approximation as

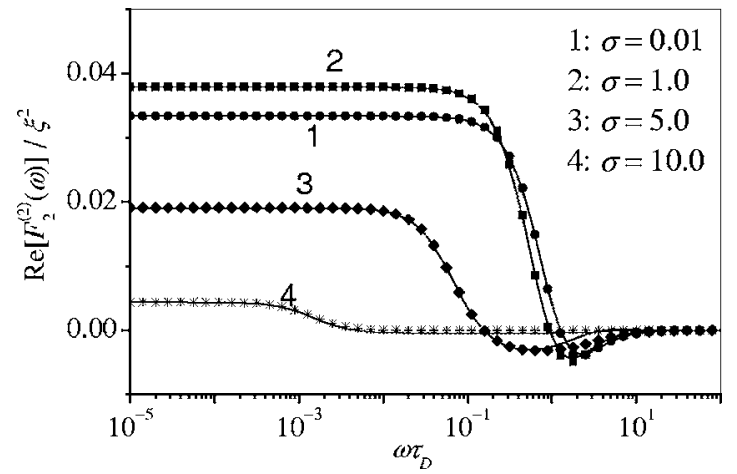

FIG. 3. Exact $\operatorname{Re}\left[F_{2}^{(2)}(\omega)\right] / \xi^{2}$ [Eq. (12), solid lines] and approximate [Eq. (16), symbols] solutions. 


$$
1-i \omega \widetilde{\Phi}^{(1)}(\omega)=\frac{\Delta_{2}}{1+i \omega / \lambda_{1}}+\frac{1-\Delta_{2}}{1+i \omega \tau_{W}^{2}} .
$$

Here $\Delta_{2}$ and $\tau_{W}^{2}$ may be evaluated from Eq. (10) using $\lambda_{1}$, $\tau_{m}=\tau$, and $\tau_{m}^{e f f}=\tau^{e f f}$ (see Table I). For $\sigma<1$ and $\sigma \gg 1$, their behavior is $\Delta_{2}=1+\sigma / 35+\cdots, \tau_{W}^{2} / \tau_{D}=\sigma / 70+\cdots$ and $\Delta_{2}=1$ $+\sigma^{-1}+\cdots, \tau_{W}^{2} / \tau_{D} \sim-1 / 2 \sigma+\cdots$, respectively. Thus, setting $\xi(t)=\xi \cos \omega t$, Eq. (14) yields

$$
\begin{aligned}
f_{2}^{(2)}(t)= & \frac{\chi_{2} \xi^{2}}{2} \operatorname{Re}\left[\left(1+\frac{e^{2 i \omega t}}{1+2 i \omega \tau_{2}^{\text {eff }}}\right)\right. \\
& \left.\times\left(\frac{\Delta_{2}}{1+i \omega / \lambda_{1}}+\frac{1-\Delta_{2}}{1+i \omega \tau_{W}^{2}}\right)\right],
\end{aligned}
$$

Apparently, the KER calculated from the approximate Eq. (16) is in excellent agreement with the exact Eq. (12); see Figs. 2 and 3. The results suggest a method of measuring the overbarrier relaxation time $1 / \lambda_{1}$, i.e., the inverse Kramers escape rate, using the dc component of the Kerr response. For free diffusion $(\sigma=0), \chi_{2}=1 / 15, \tau_{2}^{e f f}=\tau_{D} / 3, \lambda_{1}=1 / \tau_{D}$, and $\Delta_{2}=1$ so that Eq. (16) reduces to the known results $[3,21]$.

Finally as in $[3,21], f_{1}^{(1)}(t)$ and $f_{2}^{(2)}(t)$ yield the NLDE $f_{1}^{(3)}(t)$. We have

$$
\begin{aligned}
f_{1}^{(3)}(t)= & -\frac{1}{6}\left(\left\langle P_{1}^{4}\right\rangle_{0}-3\left\langle P_{1}^{2}\right\rangle_{0}^{2}\right) \int_{-\infty}^{t} \xi\left(t^{\prime}\right) \dot{\Phi}^{(3)}\left(t-t^{\prime}\right) \\
& \times \int_{-\infty}^{t^{\prime}} \xi\left(t^{\prime \prime}\right) \dot{\Phi}_{2,2}\left(t^{\prime}-t^{\prime \prime}\right) \\
& \times \int_{-\infty}^{t^{\prime \prime}} \dot{\Phi}^{(1)}\left(t^{\prime \prime}-t^{\prime \prime \prime}\right) \xi\left(t^{\prime \prime \prime}\right) d t^{\prime \prime \prime} d t^{\prime \prime} d t^{\prime},
\end{aligned}
$$

representing the generalization of Eq. (21) of Ref. [3] or Eq. (14.21) of Ref. [21] to a mean field. $\Phi^{(3)}(t)$ contains the contribution of the matrix elements of the KER to $f_{1}^{(3)}(t)$ and is represented by a two-mode approximation as the propagator involves overbarrier relaxation.

In this paper, we have described exact and approximate calculations of the nonlinear orientational ac response of permanent dipoles in the presence of a uniaxial potential Eq. (1). The approximate calculation accurately represents the relevant matrix elements of the exact time ordered matrix exponential solution generated by perturbation theory using Picard's method $[3,21,22]$. Thus the approximate solution effectively generalizes the existing analytic results for noninteracting dipoles in ac driving fields to a mean field potential and has a similar mathematical form Eq. (16) (but with parameters given in terms of the barrier height parameter $\sigma$ ) so explaining the successful application of the known frequency dependence of the KER for free diffusion to the analysis of experimental spectra of electric birefringence of nematics which was previously done without any theoretical justification (see, e.g., Ref. [23]). The results apply both to nonlinear dielectric relaxation and KER of nematics and also to magnetic birefringence relaxation of ferrofluids. The solution of the problem citing, for example, the matrix element $f_{2}^{(2)}(t)$, clearly demonstrates that the dc component of a second order nonlinear response contains information about the linear response function. This fact suggests possible methods of measurement of the overbarrier relaxation time (inverse Kramers rate) via the dc electric or magnetic birefringence. We have illustrated the calculation for the simplest mean field potential and have ignored induced moments. The calculation may, however, be very easily extended to (a) nonstationary response, (b) induced moments, and (c) other mean field potentials such as biaxial anisotropy. Finally, the method may be extended to fractional Brownian motion resulting in anomalous relaxation as described in Ref. [6].

The HEA Ireland PRTLI Nanomaterials Initiative is thanked for financial support.
[1] P. Debye, Polar Molecules (Chem. Catalog Co., New York, 1929).

[2] Yu. L. Raikher and V. I. Stepanov, Adv. Chem. Phys. 129, 419 (2004).

[3] W. T. Coffey and B. V. Paranjape, Proc. R. Ir. Acad., Sect. A 78, 17 (1978).

[4] A. Morita and S. Watanabe, Adv. Chem. Phys. 56, 255 (1984).

[5] J. L. Déjardin et al., Adv. Chem. Phys. 117, 275 (2001).

[6] W. T. Coffey et al., The Langevin Equation, 2nd ed. (World Scientific, Singapore, 2004).

[7] K. De Smet et al., Phys. Rev. E 57, 1384 (1998).

[8] H. Benoit, Ann. Phys. (Paris) 6, 561 (1951).

[9] T. Furukawa and K. Matsumoto, Jpn. J. Appl. Phys., Part 1 31, 840 (1992)

[10] A. J. Martin et al., Symp. Faraday Soc. 5, 119 (1971).

[11] P. L. Nordio and P. Busolin. J. Chem. Phys. 55, 5485 (1971).

[12] B. A. Storonkin, Kristallografiya 30, 841 (1985) [Sov. Phys.
Crystallogr.. 30, 489 (1985)].

[13] W. T. Coffey et al., Physica A 213, 551 (1995).

[14] R. Kubo et al., Statistical Physics II: Non-Equilibrium Statistical Mechanics, 2nd ed. (Springer-Verlag, Berlin, 1991).

[15] Y. L. Raikher et al., J. Colloid Interface Sci. 144, 308 (1991).

[16] B. U. Felderhof and R. B. Jones, J. Chem. Phys. 115, 7852 (2001); R. B. Jones, J. Phys.: Condens. Matter 14, 7719 (2002).

[17] Y. L. Raikher and V. I. Stepanov, Phys. Rev. B 66, 214406 (2002).

[18] W. F. Brown, Jr., Phys. Rev. 130, 677 (1963).

[19] A. Morita, Phys. Rev. A 34, 1499 (1986).

[20] A. Szabo, J. Chem. Phys. 72, 4620 (1980).

[21] W. T. Coffey. Adv. Chem. Phys. 63, 69 (1985).

[22] K. Hosokawa et al., J. Chem. Phys. 110, 4101 (1999).

[23] M. Schadt, J. Chem. Phys. 67, 210 (1977). 